International Journal of Pure and Applied Mathematics

Volume 97 No. 4 2014, 527-541

ISSN: 1311-8080 (printed version); ISSN: 1314-3395 (on-line version)

url: http://www.ijpam.eu

doi: http://dx.doi.org/10.12732/ijpam.v97i4.12

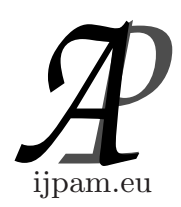

\title{
SOME PROPERTIES OF STARLIKE AND CLOSE TO CONVEX FUNCTIONS WITH RESPECT TO SYMMETRIC CONJUGATE POINTS
}

\author{
C. Selvaraj ${ }^{1}$, G. Thirupathi ${ }^{2} \S$ \\ ${ }^{1}$ Presidency College \\ Chennai, 600 005, Tamilnadu, INDIA \\ ${ }^{2}$ R.M.K. Engineering College \\ R.S.M. Nagar, Kavaraipettai, 601 206, Tamilnadu, INDIA
}

\begin{abstract}
In the present paper, we introduce and investigate some new subclasses of $\alpha$ - starlike and $\alpha$ - close to convex functions with respect to symmetric conjugate points. Inclusion relationships, integral representations and some interesting convolution properties for these functions are obtained.
\end{abstract}

AMS Subject Classification: 30C45

Key Words: multivalent functions, hadamard product, close to convex functions, $(2 j, k)$ symmetric conjugate points

\section{Introduction, Definitions and Preliminaries}

Let $\mathcal{A}_{p}$ be the class of functions $f(z)$, of the form

$$
f(z)=z^{p}+\sum_{n=p+1}^{\infty} a_{n} z^{n}
$$

which are analytic in the unit disc $\mathbb{U}=\{z \in \mathbb{C}:|z|<1\}$. And let $\mathcal{A}=\mathcal{A}_{1}$.

We denote by $\mathcal{S}^{*}, \mathcal{C}, \mathcal{K}$ and $\mathcal{C}^{*}$ the familiar subclasses of $\mathcal{A}$ consisting of

Received: August 30, 2014

(C) 2014 Academic Publications, Ltd.

$\S_{\text {Correspondence author }}$ url: www.acadpubl.eu 
functions which are respectively starlike, convex, close-to-convex and quasiconvex in $\mathbb{U}$. The references of the field are $[5,6]$, it covers most of the topics.

Let $\mathcal{S}$ be the subclass of $\mathcal{A}$ consisting of all functions which are univalent in $\mathbb{U}$. Also, let $\mathcal{P}$ denote the class of functions of the form

$$
p(z)=1+\sum_{n=1}^{\infty} c_{n} z^{n}
$$

which are analytic and convex in $\mathbb{U}$ and satisfy the condition

$$
\Re(p(z))>0,(z \in \mathbb{U}) .
$$

Let $f(z)$ and $g(z)$ be analytic in $\mathbb{U}$. Then we say that the function $f(z)$ is subordinate to $g(z)$ in $\mathbb{U}$, if there exists an analytic function $w(z)$ in $\mathbb{U}$ such that $|w(z)|<|z|$ and $f(z)=g(w(z))$, denoted by $f(z) \prec g(z)$. If $g(z)$ is univalent in $\mathbb{U}$, then the subordination is equivalent to $f(0)=g(0)$ and $f(\mathbb{U}) \subset g(\mathbb{U})$.

Let $k$ be a positive integer and $j=0,1,2, \ldots(k-1)$. A domain $D$ is said to be $(j, k)$-fold symmetric if a rotation of $D$ about the origin through an angle $2 \pi j / k$ carries $D$ onto itself. A function $f \in \mathcal{A}$ is said to be $(j, k)$-symmetrical if for each $z \in \mathbb{U}$

$$
f(\varepsilon z)=\varepsilon^{j} f(z)
$$

where $\varepsilon=\exp (2 \pi i / k)$. The family of $(j, k)$-symmetrical functions will be denoted by $\mathcal{F}_{k}^{j}$. For every function $f$ defined on a symmetrical subset $\mathbb{U}$ of $\mathbb{C}$, there exits a unique sequence of $(j, k)$-symmetrical functions $f_{j, k}(z), j=$ $0,1, \ldots, k-1$ such that

$$
f=\sum_{j=0}^{k-1} f_{j, k}
$$

Moreover,

$$
f_{j, k}(z)=\frac{1}{k} \sum_{\nu=0}^{k-1} \frac{f\left(\varepsilon^{\nu} z\right)}{\varepsilon^{\nu p j}}, \quad\left(f \in \mathcal{A}_{p} ; k=1,2, \ldots ; j=0,1,2, \ldots(k-1)\right)
$$

This decomposition is a generalization of the well known fact that each function defined on a symmetrical subset $\mathbb{U}$ of $\mathbb{C}$ can be uniquely represented as the sum of an even function and an odd functions (see Theorem 1 of [7]). It is obvious that $f_{j, k}(z)$ is a linear operator from $\mathbb{U}$ into $\mathbb{U}$. The notion of $(j, k)$-symmetrical functions was first introduced and studied by P. Liczberski and J. Polubiński in [7]. The class of $(j, k)$-symmetrical functions was extended 
to the class $(j, k)$-symmetrical conjugate functions in [12]. For fixed positive integers $j$ and $k$, let $f_{2 j, k}(z)$ be defined by the following equality

$$
f_{2 j, k}(z)=\frac{1}{2 k} \sum_{\nu=0}^{k-1}\left[\varepsilon^{-\nu p j} f\left(\varepsilon^{\nu} z\right)+\varepsilon^{\nu p j} \overline{f\left(\varepsilon^{\nu} \bar{z}\right)}\right], \quad\left(f \in \mathcal{A}_{p}\right) .
$$

If $\nu$ is an integer, then the following identities follow directly from (1.4):

$$
\begin{array}{r}
f_{2 j, k}^{\prime}(z)=\frac{1}{2 k} \sum_{\nu=0}^{k-1}\left[\varepsilon^{-\nu p j+\nu} f^{\prime}\left(\varepsilon^{\nu} z\right)+\varepsilon^{\nu p j-\nu} \overline{f^{\prime}\left(\varepsilon^{\nu} \bar{z}\right)}\right], \\
f_{2 j, k}^{\prime \prime}(z)=\frac{1}{2 k} \sum_{\nu=0}^{k-1}\left[\varepsilon^{-\nu p j+2 \nu} f^{\prime \prime}\left(\varepsilon^{\nu} z\right)+\varepsilon^{\nu p j-2 \nu} \overline{f^{\prime \prime}\left(\varepsilon^{\nu} \bar{z}\right)}\right],
\end{array}
$$

and

$$
\begin{aligned}
& f_{2 j, k}\left(\varepsilon^{\nu} z\right)=\varepsilon^{\nu p j} f_{2 j, k}(z), \quad f_{2 j, k}(z)=\overline{f_{2 j, k}(\bar{z})}, \\
& f_{2 j, k}^{\prime}\left(\varepsilon^{\nu} z\right)=\varepsilon^{\nu p j-\nu} f_{2 j, k}^{\prime}(z), \quad f_{2 j, k}^{\prime}(\bar{z})=\overline{f_{2 j, k}^{\prime}(z)} .
\end{aligned}
$$

Motivated by the concept introduced by Sakaguchi in [11], recently several subclasses of analytic functions with respect to $k$-symmetric points were introduced and studied by various authors (see $[1,2,14,15,16])$. Parvatham in ([10]) introduced and investigated $K_{n}(\alpha, h)$ - so called class of $\alpha$ starlike functions with respect to $n$ symmetric points.

Definition 1.1. The function $f \in \mathcal{A}_{p}$ and $\frac{f(z) f^{\prime}(z)}{z} \neq 0$ in $\mathbb{U}$ is said to be in the class $\mathcal{S}_{p}^{j, k}(\alpha, \phi)$ if and only if it satisfies the condition

$$
\frac{1}{p}\left(\frac{\alpha z\left(z f^{\prime}(z)\right)^{\prime}+(1-\alpha) z f^{\prime}(z)}{\alpha z f_{2 j, k}^{\prime}(z)+(1-\alpha) f_{2 j, k}(z)}\right) \prec \phi(z), \quad(z \in \mathbb{U}),
$$

where, $\phi \in \mathcal{P}, 0 \leq \alpha \leq 1$ and $f_{2 j, k}(z) \neq 0$ is defined by the equality (1.4). Similarly, we say that a function $f \in \mathcal{A}_{p}$ is in the class $\mathcal{C}_{p}^{j, k}(\alpha, \phi)$ if and only if

$$
z f^{\prime} \in \mathcal{S}_{p}^{j, k}(\alpha, \phi)
$$

Definition 1.2. The function $f \in \mathcal{A}_{p}$ and $\frac{f(z) f^{\prime}(z)}{z} \neq 0$ in $\mathbb{U}$ is said to be in the class $\mathcal{M}_{p}^{j, k}(\alpha, \phi)$ if and only if it satisfies the condition

$$
\frac{1}{p}\left((1-\alpha) \frac{z f^{\prime}(z)}{f_{2 j, k}(z)}+\alpha \frac{\left(z f^{\prime}(z)\right)^{\prime}}{f_{2 j, k}^{\prime}(z)}\right) \prec \phi(z), \quad(z \in \mathbb{U}),
$$

where, $\phi \in \mathcal{P}$ and $\alpha \geq 0$. 
Definition 1.3. The function $f \in \mathcal{A}_{p}$ and $\frac{f(z) f^{\prime}(z)}{z} \neq 0$ in $\mathbb{U}$ is said to be in the class $\mathcal{K}_{p}^{j, k}(\alpha, \phi)$ if and only if it satisfies the condition

$$
\frac{1}{p}\left(\frac{\alpha z\left(z f^{\prime}(z)\right)^{\prime}+(1-\alpha) z f^{\prime}(z)}{\alpha z g_{2 j, k}^{\prime}(z)+(1-\alpha) g_{2 j, k}(z)}\right) \prec \phi(z), \quad(z \in \mathbb{U}),
$$

where, $\phi \in \mathcal{P}, 0 \leq \alpha \leq 1$ and $g_{2 j, k}(z) \neq 0$ is defined by (1.4) with $g_{2 j, k}(z) \in$ $\mathcal{S}_{p}^{j, k}(\alpha, \phi)$.

Remark 1.1. In view of definitions 1.1 and 1.2, we know that the classes $\mathcal{S}_{p}^{j, k}(\alpha, \phi)$ and $\mathcal{C}_{p}^{j, k}(\alpha, \phi)$ unify the classes of $\alpha$ starlike and $\alpha$ convex functions with respect to $(2 j, k)$ symmetric conjugate points. Furhter we note that several other new and well known subclasses of analytic functions can be obtained as special cases of $\mathcal{S}_{p}^{j, k}(\alpha, \phi)$ and $\mathcal{C}_{p}^{j, k}(\alpha, \phi)$.

If we put $\alpha=0$, then the classes $\mathcal{S}_{p}^{j, k}(\alpha, \phi)$ and $\mathcal{C}_{p}^{j, k}(\alpha, \phi)$ reduces to the respective class $\mathcal{S}_{p}^{j, k}(\phi)$ and $\mathcal{C}_{p}^{j, k}(\phi)$ introduced by Karthikeyan [12]. Also, if we let $p=j=k=1, \alpha=0$ and $h(z)=\frac{1+z}{1-z}$ then the class $\mathcal{S}_{p}^{j, k}(\alpha, \phi)$ reduces to the class $\mathcal{S}_{c}^{*}$ investigated by EL Ashwa and Thomas in [4].

Lemma 1.1. $[3,8]$ Let $\beta, \gamma \in \mathbb{C}$. Suppose that $\phi$ is convex and univalent in $\mathbb{U}$ with $\phi(0)=1$ and $\Re(\beta \phi(z)+\gamma)>0,(z \in \mathbb{U})$ and let $p$ be analytic in $\mathbb{U}$ with $p(0)=1$, then the subordination

$$
p(z)+\frac{z p^{\prime}(z)}{\beta p(z)+\gamma} \prec \phi(z) \Rightarrow p(z) \prec \phi(z) .
$$

Lemma 1.2. [9] Let $\beta, \gamma \in \mathbb{C}$. Suppose that $\phi$ is convex and univalent in $\mathbb{U}$ with $\phi(0)=1$ and $\Re(\beta \phi(z)+\gamma)>0,(z \in \mathbb{U})$. Also, let $q(z) \prec \phi(z)$. If $u \in \mathcal{P}$ and satisfies the subordination

$$
u(z)+\frac{z u^{\prime}(z)}{\beta q(z)+\gamma} \prec \phi(z),
$$

then,

$$
u(z) \prec \phi(z) .
$$

Lemma 1.3. [13] Let $\phi \in \mathcal{P}$. Then,

$$
\mathcal{S}_{p}^{j, k}(\phi) \subset \mathcal{C} \subset \mathcal{S} .
$$

Lemma 1.4. [13] Let $\phi \in \mathcal{P}$. Then,

$$
\mathcal{C}_{p}^{j, k}(\phi) \subset \mathcal{C}^{*} \subset \mathcal{C} .
$$




\section{Inclusion Relationship}

Theorem 2.1. Let $\phi \in \mathcal{P}, 0 \leq \alpha \leq 1$ and let $f \in \mathcal{S}_{p}^{j, k}(\alpha, \phi)$. Then $f_{2 j, k} \in \mathcal{S}_{p}^{j, k}(\alpha, \phi)$. Furthermore for any $z \in \mathbb{U}$,

$$
\mathcal{S}_{p}^{j, k}(\alpha, \phi) \subset \mathcal{S}_{p}^{j, k}(\phi) \subset \mathcal{C} \subset \mathcal{S} .
$$

Proof. Let $f \in \mathcal{S}_{p}^{j, k}(\alpha, \phi)$. From the definition of $\mathcal{S}_{p}^{j, k}(\alpha, \phi)$, we have

$$
\frac{1}{p}\left(\frac{\alpha z\left(z f^{\prime}(z)\right)^{\prime}+(1-\alpha) z f^{\prime}(z)}{\alpha z f_{2 j, k}^{\prime}(z)+(1-\alpha) f_{2 j, k}(z)}\right) \prec \phi(z), \quad(z \in \mathbb{U}) .
$$

On a simple computation, we have

$$
\frac{1}{p}\left(\frac{z f^{\prime}(z)+\alpha z^{2} f^{\prime \prime}(z)}{(1-\alpha) f_{2 j, k}(z)+\alpha z f_{2 j, k}^{\prime}(z)}\right) \prec \phi(z) .
$$

If we replace $z$ by $\varepsilon^{\nu} z$ in (2.2), then (2.2) will be of the form

$$
\begin{aligned}
\frac{1}{p}\left(\frac{\varepsilon^{\nu} z f^{\prime}\left(\varepsilon^{\nu} z\right)+\alpha\left(\varepsilon^{\nu} z\right)^{2} f^{\prime \prime}\left(\varepsilon^{\nu} z\right)}{(1-\alpha) f_{2 j, k}\left(\varepsilon^{\nu} z\right)+\alpha z \varepsilon^{\nu} f_{2 j, k}^{\prime}\left(\varepsilon^{\nu} z\right)}\right) & \prec \phi(z) \\
& (z \in \mathcal{U} ; \nu=0,1,2, \ldots, k-1) .
\end{aligned}
$$

Also we have,

$$
\begin{aligned}
\frac{1}{p}\left(\frac{\overline{\varepsilon^{\nu} \bar{z}} \overline{f^{\prime}\left(\varepsilon^{\nu} \bar{z}\right)}+\alpha \overline{\left(\varepsilon^{\nu} \bar{z}\right)^{2}} \overline{f^{\prime \prime}\left(\varepsilon^{\nu} \bar{z}\right)}}{(1-\alpha) \overline{f_{2 j, k}\left(\varepsilon^{\nu} \bar{z}\right)}+\alpha \overline{z \varepsilon^{\nu}} \overline{f_{2 j, k}^{\prime}\left(\varepsilon^{\nu} \bar{z}\right)}}\right) \prec \phi(z) \\
\qquad(z \in \mathcal{U} ; \nu=0,1,2, \ldots, k-1) .
\end{aligned}
$$

Using the equality (1.6), (2.3) and (2.4) can be rewritten as

$$
\frac{1}{p}\left(\frac{\varepsilon^{\nu-\nu p j}\left(z f^{\prime}\left(\varepsilon^{\nu} z\right)+\alpha z^{2} \varepsilon^{\nu} f^{\prime \prime}\left(\varepsilon^{\nu} z\right)\right)}{(1-\alpha) f_{2 j, k}(z)+\alpha z f_{2 j, k}^{\prime}(z)}\right)^{\prec \phi(z)}
$$

and

$$
\frac{1}{p}\left(\frac{\varepsilon^{-\nu+\nu p j}\left(z \overline{f^{\prime}\left(\varepsilon^{\nu} \bar{z}\right)}+\alpha z^{2} \varepsilon^{-\nu} \overline{f^{\prime \prime}\left(\varepsilon^{\nu} \bar{z}\right)}\right)}{(1-\alpha) f_{2 j, k}(z)+\alpha z f_{2 j, k}^{\prime}(z)}\right)^{\prec \phi(z)}
$$




$$
(z \in \mathcal{U} ; \nu=0,1,2, \ldots, k-1)
$$

Adding (2.5) and (2.6), we get

$$
\begin{gathered}
\frac{1}{p}\left(\frac{\frac{z}{2}\left(\varepsilon^{\nu-\nu p j} f^{\prime}\left(\varepsilon^{\nu} z\right)+\varepsilon^{-\nu+\nu p j} \overline{f^{\prime}\left(\varepsilon^{\nu} \bar{z}\right)}\right)+\frac{\alpha z^{2}}{2}\left(\varepsilon^{2 \nu-\nu p j} f^{\prime \prime}\left(\varepsilon^{\nu} z\right)+\varepsilon^{-2 \nu+\nu p j} \overline{f^{\prime \prime}\left(\varepsilon^{\nu} \bar{z}\right)}\right)}{(1-\alpha) f_{2 j, k}(z)+\alpha z f_{2 j, k}^{\prime}(z)}\right) \\
\prec \phi(z) .
\end{gathered}
$$

Let $\nu=0,1,2, \ldots, k-1$ in $(2.7)$ respectively and summing them, we get

$$
\begin{aligned}
& \frac{1}{p} \frac{\frac{z}{2 k} \sum_{\nu=0}^{k-1}\left[\varepsilon^{\nu-\nu p j} f^{\prime}\left(\varepsilon^{\nu} z\right)+\varepsilon^{-\nu+\nu p j} \overline{f^{\prime}\left(\varepsilon^{\nu} \bar{z}\right)}\right]+\frac{\alpha z^{2}}{2 k} \sum_{\nu=0}^{k-1}\left[\varepsilon^{2 \nu-\nu p j} f^{\prime \prime}\left(\varepsilon^{\nu} z\right)+\varepsilon^{-2 \nu+\nu p j} \overline{f^{\prime \prime}\left(\varepsilon^{\nu} \bar{z}\right)}\right]}{(1-\alpha) f_{2 j, k}(z)+\alpha z f_{2 j, k}^{\prime}(z)} \\
& \prec \phi(z),
\end{aligned}
$$

or equivalently,

$$
\frac{1}{p} \frac{z f_{2 j, k}^{\prime}(z)+\alpha z^{2} f_{2 j, k}^{\prime \prime}(z)}{(1-\alpha) f_{2 j, k}(z)+\alpha z f_{2 j, k}^{\prime}(z)} \prec \phi(z)
$$

which implies that $f_{2 j, k} \in \mathcal{S}_{p}^{j, k}(\alpha, \phi)$.

If we set

$$
p(z)=\frac{z f_{2 j, k}^{\prime}(z)}{p f_{2 j, k}(z)}, \quad(z \in \mathbb{U})
$$

then $(2.9)$ can be written as

$$
\frac{1}{p} \frac{z f_{2 j, k}^{\prime}(z)+\alpha z^{2} f_{2 j, k}^{\prime \prime}(z)}{(1-\alpha) f_{2 j, k}(z)+\alpha z f_{2 j, k}^{\prime}(z)}=p(z)+\frac{\alpha z p^{\prime}(z)}{(1-\alpha)+p \alpha p(z)} \prec \phi(z) .
$$

It is very clear that $p(z) \prec \phi(z)$, since $\alpha$ is a real number with the condition imposed $0 \leq \alpha \leq 1$.

Setting

$$
h(z)=\frac{z f^{\prime}(z)}{p f_{2 j, k}(z)}, \quad(z \in \mathbb{U})
$$


Now

$$
\begin{aligned}
& \frac{1}{p}\left(\frac{(1-\alpha) z f^{\prime}(z)+\alpha z\left(z f^{\prime}(z)\right)^{\prime}}{(1-\alpha) f_{2 j, k}(z)+\alpha z f_{2 j, k}^{\prime}(z)}\right) \\
= & \frac{1}{p} \frac{(1-\alpha) p h(z) f_{2 j, k}(z)+\alpha z p\left[h^{\prime}(z) f_{2 j, k}(z)+h(z) f_{2 j, k}^{\prime}(z)\right]}{(1-\alpha) f_{2 j, k}(z)+\alpha z f_{2 j, k}^{\prime}(z)} \\
= & \frac{(1-\alpha) h(z)+\alpha z h^{\prime}(z)+\frac{\alpha z f_{2 j, k}^{\prime}(z)}{f_{2 j, k}(z)} \cdot h(z)}{(1-\alpha)+\frac{\alpha z f_{2 j, k}^{\prime}(z)}{f_{2 j, k}(z)}} \\
= & h(z)+\frac{\alpha z h^{\prime}(z)}{(1-\alpha)+p \alpha p(z)} .
\end{aligned}
$$

Now, by applying the above proof for $p(z) \prec \phi(z)$ and using Lemma 1.2 in (2.11), we know that

$$
h(z)=\frac{z f^{\prime}(z)}{p f_{2 j, k}(z)} \prec \phi(z), \quad(z \in \mathbb{U}),
$$

which implies that

$$
\mathcal{S}_{p}^{j, k}(\alpha, \phi) \subset \mathcal{S}_{p}^{j, k}(\phi)
$$

And also

$$
\mathcal{S}_{p}^{j, k}(\alpha, \phi) \subset \mathcal{S}_{p}^{j, k}(\phi) \subset \mathcal{C} \subset \mathcal{S}
$$

By means of Lemma 1.4 and making use of similar arguments given in the proof for Theorem 2.1, we easily get the following inclusion relationship for the class $\mathcal{C}_{p}^{j, k}(\alpha, \phi)$.

Corollary 2.2. Let $\phi \in \mathcal{P}$ and $0 \leq \alpha \leq 1$, then

$$
\mathcal{C}_{p}^{j, k}(\alpha, \phi) \subset \mathcal{C}_{p}^{j, k}(\phi) \subset \mathcal{C}^{*} \subset \mathcal{C}
$$

Theorem 2.3. Let $\phi \in \mathcal{P}$ and $0 \leq \alpha \leq 1$, then

$$
\mathcal{K}_{p}^{j, k}(\alpha, \phi ; g) \subset \mathcal{K}_{p}^{j, k}(\phi ; g)
$$


Proof. Let $f \in \mathcal{K}_{p}^{j, k}(\alpha, \phi)$. Setting $p(z)=\frac{z f^{\prime}(z)}{p g_{2 j, k}(z)}$ and $q(z)=\frac{z g_{2 j, k}^{\prime}(z)}{p g_{2 j, k}(z)}$, we have,

$$
\begin{aligned}
\frac{1}{p} \frac{\alpha z\left(z f^{\prime}(z)\right)^{\prime}+(1-\alpha) z f^{\prime}(z)}{\alpha z g_{2 j, k}^{\prime}(z)+(1-\alpha) g_{2 j, k}(z)} & =\frac{\alpha z p^{\prime}(z)+p(z)[(1-\alpha)+\alpha q(z)]}{(1-\alpha)+p \alpha q(z)} \\
& =p(z)+\frac{\alpha z p^{\prime}(z)}{(1-\alpha)+p \alpha q(z)} \prec \phi(z)
\end{aligned}
$$

since $f \in \mathcal{K}_{p}^{j, k}(\alpha, \phi)$. Here $q(z) \prec \phi(z)$ (by lemma). Again an application of Lemma (1.2) yields $p(z)=\frac{z f(z)}{p g_{2 j, k}(z)}$ which estabilish the theorem.

Theorem 2.4. Let $\phi \in \mathcal{P}$ and $0 \leq \alpha \leq 1$, then

$$
\mathcal{M}_{p}^{j, k}(\alpha, \phi) \subset \mathcal{S}_{p}^{j, k}(\phi) \subset \mathcal{C} \subset \mathcal{S} .
$$

Proof. Suppose that $f \in \mathcal{M}_{p}^{j, k}(\alpha, \phi)$. It follows form (1.8) that

$$
\begin{aligned}
\frac{1}{p}\left((1-\alpha) \frac{z f^{\prime}(z)}{f_{2 j, k}(z)}+\right. & \left.\alpha \frac{\left(z f^{\prime}(z)\right)^{\prime}}{f_{2 j, k}^{\prime}(z)}\right) \\
& =\frac{(1-\alpha)}{p} \frac{z f^{\prime}(z)}{f_{2 j, k}(z)}+\frac{\alpha}{p} \frac{f^{\prime}(z)+z f^{\prime \prime}(z)}{f_{2 j, k}^{\prime}(z)} \prec \phi(z)
\end{aligned}
$$

If we replace $z$ by $\varepsilon^{\nu} z(\nu=0,1,2, \ldots k-1)$ in $(2.12)$, then $(2.12)$ will be of the form

$$
\frac{(1-\alpha)}{p} \frac{\varepsilon^{\nu} z f^{\prime}\left(\varepsilon^{\nu} z\right)}{f_{2 j, k}\left(\varepsilon^{\nu} z\right)}+\frac{\alpha}{p} \frac{f^{\prime}\left(\varepsilon^{\nu} z\right)+\varepsilon^{\nu} z f^{\prime \prime}\left(\varepsilon^{\nu} z\right)}{f_{2 j, k}^{\prime}\left(\varepsilon^{\nu} z\right)} \prec \phi(z) .
$$

From (2.13), we have,

$$
\frac{(1-\alpha)}{p} \frac{\overline{\varepsilon^{\nu} \bar{z}} \overline{f^{\prime}\left(\varepsilon^{\nu} \bar{z}\right)}}{\overline{f_{2 j, k}\left(\varepsilon^{\nu} \bar{z}\right)}}+\frac{\alpha}{p} \frac{\overline{f^{\prime}\left(\varepsilon^{\nu} \bar{z}\right)}+\overline{\varepsilon^{\nu} \bar{z}} \overline{f^{\prime \prime}\left(\varepsilon^{\nu} \bar{z}\right)}}{\overline{f_{2 j, k}^{\prime}\left(\varepsilon^{\nu} \bar{z}\right)}} \prec \phi(z) .
$$

Proceeding as in Theorem (2.1), we have

$$
\frac{1}{p}\left((1-\alpha) \frac{z f_{2 j, k}^{\prime}(z)}{f_{2 j, k}(z)}+\alpha \frac{\left(z f_{2 j, k}^{\prime}(z)\right)^{\prime}}{f_{2 j, k}^{\prime}(z)}\right) \prec \phi(z) .
$$

Letting 


$$
p(z)=\frac{z f_{2 j, k}^{\prime}(z)}{p f_{2 j, k}(z)}, \quad(z \in \mathbb{U}) .
$$

Then, (2.15) can be written as

$$
\frac{1}{p}\left((1-\alpha) \frac{z f_{2 j, k}^{\prime}(z)}{f_{2 j, k}(z)}+\alpha \frac{\left(z f_{2 j, k}^{\prime}(z)\right)^{\prime}}{f_{2 j, k}^{\prime}(z)}\right)=p(z)+\alpha \frac{z p^{\prime}(z)}{p p(z)} \prec \phi(z) .
$$

By Lemma 1.1, we have

$$
p(z)=\frac{z f_{2 j, k}^{\prime}(z)}{p f_{2 j, k}(z)} \prec \phi(z) .
$$

If we let

$$
h(z)=\frac{z f^{\prime}(z)}{p f_{2 j, k}(z)},
$$

then (1.8), can be written as follows:

$$
\frac{1}{p}\left((1-\alpha) \frac{z f^{\prime}(z)}{f_{2 j, k}(z)}+\alpha \frac{\left(z f^{\prime}(z)\right)^{\prime}}{f_{2 j, k}^{\prime}(z)}\right)=h(z)+\alpha \frac{z h^{\prime}(z)}{p p(z)} \prec \phi(z) .
$$

Since

$$
p(z) \prec \phi(z),
$$

By Lemma 1.2, yields

$$
\mathcal{M}_{p}^{j, k}(\alpha, \phi) \subset \mathcal{S}_{p}^{j, k}(\phi) .
$$

Then Lemma 1.3, we have

$$
\mathcal{M}_{p}^{j, k}(\alpha, \phi) \subset \mathcal{S}_{p}^{j, k}(\phi) \subset \mathcal{C} \subset \mathcal{S} .
$$




\section{Integral Representation}

Theorem 3.1. Let $f \in \mathcal{S}_{p}^{j, k}(\alpha, \phi)$ with $0<\alpha \leq 1$. Then

$$
\begin{aligned}
& f_{2 j, k}(z)= \\
& \frac{1}{\alpha} z^{1-\frac{1}{\alpha}} \int_{0}^{z} \exp \left(\frac{p}{2 k} \sum_{\nu=0}^{k-1} \int_{0}^{u} \frac{\phi\left(w\left(\varepsilon^{\nu} \zeta\right)\right)+\overline{\phi\left(w\left(\varepsilon^{\nu} \bar{\zeta}\right)\right)}-2}{\zeta} d \zeta\right) u^{\frac{1}{\alpha}+p-2} d u
\end{aligned}
$$

where, $f_{2 j, k}(z)$ is defined by (1.4), $w$ is analytic in $\mathbb{U}$ with

$$
w(0)=0 \text { and }|w(z)|<1, \quad(z \in \mathbb{U}) .
$$

Proof. Suppose that $f \in \mathcal{S}_{p}^{j, k}(\alpha, \phi)$. We know that the condition (1.7) can be written as

$$
\frac{1}{p}\left(\frac{\alpha z\left(z f^{\prime}(z)\right)^{\prime}+(1-\alpha) z f^{\prime}(z)}{\alpha z f_{2 j, k}^{\prime}(z)+(1-\alpha) f_{2 j, k}(z)}\right)=\phi(w(z)), \quad(z \in \mathbb{U})
$$

where, $w$ is analytic in $\mathbb{U}$ with

$$
w(0)=0 \text { and }|w(z)|<1, \quad(z \in \mathbb{U}) .
$$

By similar application of the arguments given in the proof for Theorem 2.1 to (3.2), we get,

$$
\begin{aligned}
& \frac{1}{p} \frac{\alpha z\left(z f_{2 j, k}^{\prime}(z)\right)^{\prime}+(1-\alpha) z f_{2 j, k}^{\prime}(z)}{\alpha z f_{2 j, k}^{\prime}(z)+(1-\alpha) f_{2 j, k}(z)} \\
& \quad=\frac{1}{2 k} \sum_{\nu=0}^{k-1}\left(\phi\left(w\left(\varepsilon^{\nu} z\right)\right)+\overline{\phi\left(w\left(\varepsilon^{\nu} \bar{z}\right)\right)}\right)
\end{aligned}
$$

From (3.3),

$$
\begin{aligned}
& \frac{\alpha\left(z f_{2 j, k}^{\prime}(z)\right)^{\prime}+(1-\alpha) f_{2 j, k}^{\prime}(z)}{\alpha z f_{2 j, k}^{\prime}(z)+(1-\alpha) f_{2 j, k}(z)}-\frac{p}{z} \\
& =\frac{p}{2 k} \sum_{\nu=0}^{k-1} \frac{\left(\phi\left(w\left(\varepsilon^{\nu} z\right)\right)+\overline{\phi\left(w\left(\varepsilon^{\nu} \bar{z}\right)\right)}-2\right)}{z} .
\end{aligned}
$$

Integrating this equality, we get 


$$
\begin{aligned}
& \log \left(\frac{\alpha z f_{2 j, k}^{\prime}(z)+(1-\alpha) f_{2 j, k}(z)}{z^{p}}\right) \\
&=\frac{p}{2 k} \sum_{\nu=0}^{k-1} \int_{0}^{z} \frac{\left(\phi\left(w\left(\varepsilon^{\nu} \zeta\right)\right)+\overline{\phi\left(w\left(\varepsilon^{\nu} \bar{\zeta}\right)\right.}-2\right)}{\zeta} d \zeta,
\end{aligned}
$$

or equivalently,

$$
\begin{aligned}
\alpha z f_{2 j, k}^{\prime}(z)+ & (1-\alpha) f_{2 j, k}(z) \\
& =z^{p} \exp \left\{\frac{p}{2 k} \sum_{\nu=0}^{k-1} \int_{0}^{z} \frac{\left(\phi\left(w\left(\varepsilon^{\nu} \zeta\right)\right)+\overline{\phi\left(w\left(\varepsilon^{\nu} \bar{\zeta}\right)\right)}-2\right)}{\zeta} d \zeta\right\} .
\end{aligned}
$$

Now we can derive (3.1) from (3.5).

Theorem 3.2. Let $f \in \mathcal{S}_{p}^{j, k}(\alpha, \phi)$ with $0<\alpha \leq 1$. Then

$$
\begin{gathered}
f(z)= \\
\frac{1}{\alpha} z^{1-\frac{1}{\alpha}} \int_{0}^{z} \int_{0}^{u} \exp \left(\frac{p}{2 k} \sum_{\nu=0}^{k-1} \int_{0}^{\eta} \frac{\phi\left(w\left(\varepsilon^{\nu} \zeta\right)\right)+\overline{\phi\left(w\left(\varepsilon^{\nu} \bar{\zeta}\right)+\right)}-2}{\zeta} d \zeta\right) \phi(w(\eta)) d \eta \\
u^{\frac{1}{\alpha}+p-2} d u
\end{gathered}
$$

where, $w$ is analytic in $\mathbb{U}$ with $w(0)=0$ and $|w(z)|<1$.

Proof. Suppose that $f \in \mathcal{S}_{p}^{j, k}(\alpha, \phi)$. Then by (3.2) and (3.5),

$$
\begin{aligned}
& \alpha\left(z f^{\prime}(z)\right)^{\prime}+(1-\alpha) f^{\prime}(z)=\frac{\alpha z f_{2 j, k}^{\prime}(z)+(1-\alpha) f_{2 j, k}(z)}{z} \cdot \phi(w(z)) \\
& \quad=\exp \left\{\frac{p}{2 k} \sum_{\nu=0}^{k-1} \int_{0}^{z} \frac{\left(\phi\left(w\left(\varepsilon^{\nu} \zeta\right)\right)+\overline{\phi\left(w\left(\varepsilon^{\nu} \bar{\zeta}\right)\right)}-2\right)}{\zeta} d \zeta\right\} \phi(w(z)) .
\end{aligned}
$$

Integrating the above equality two times, will give the assertions of the theorem. 


\section{Convolution Conditions}

Let $f, g \in \mathcal{A}_{p}$, where $f(z)$ is given by (1.1) and $g(z)$ is defined by

$$
g(z)=z^{p}+\sum_{n=p+1}^{\infty} b_{n} z^{n}
$$

then the Hadamard product (or convolution) $f * g$ is defined by

$$
(f * g)(z)=z^{p}+\sum_{n=p+1}^{\infty} a_{n} b_{n} z^{n}=(g * f)(z) .
$$

We now derive some convolution properties for the function classes $\mathcal{S}_{p}^{j, k}(\alpha, \phi)$ and $\mathcal{C}_{p}^{j, k}(\alpha, \phi)$.

Theorem 4.1. Let $f \in \mathcal{S}_{p}^{j, k}(\alpha, \phi)$ if and only if

$$
\begin{gathered}
\frac{1}{z p}\left\{f *\left[(1-\alpha)\left(\frac{z}{(1-z)^{2}}-\frac{\phi\left(e^{i \theta}\right)}{2} h\right)+\alpha z\left(\frac{z}{(1-z)^{2}}-\frac{\phi\left(e^{i \theta}\right)}{2} h\right)^{\prime}\right](z)\right\} \\
-\frac{1}{z p}\left\{\phi\left(e^{i \theta}\right) \cdot \overline{f *\left(\frac{1-\alpha}{2} h+\frac{\alpha z}{2} h^{\prime}\right)(\bar{z})}\right\} \neq 0
\end{gathered}
$$

for all $z \in \mathbb{U}$ and $0 \leq \theta<2 \pi$ where $h$ is given by

$$
h(z)=\frac{1}{k} \sum_{\nu=0}^{k-1} \frac{z}{1-\varepsilon^{\nu} z} .
$$

Proof. Let $f \in \mathcal{S}_{p}^{j, k}(\alpha, \phi)$, then

$$
\frac{1}{p}\left(\frac{\alpha z\left(z f^{\prime}(z)\right)^{\prime}+(1-\alpha) z f^{\prime}(z)}{\alpha z f_{2 j, k}^{\prime}(z)+(1-\alpha) f_{2 j, k}(z)}\right) \prec \phi(z),
$$

it is equivalent to

$$
\frac{1}{p} \frac{\alpha z\left(z f^{\prime}(z)\right)^{\prime}+(1-\alpha) z f^{\prime}(z)}{\alpha z f_{2 j, k}^{\prime}(z)+(1-\alpha) f_{2 j, k}(z)} \neq \phi\left(e^{i \theta}\right)
$$

for all $z \in \mathbb{U}$ and $0 \leq \theta<2 \pi$. Now (4.3) can be written as in the form of 


$$
\begin{aligned}
\frac{1}{p}\left\{(1-\alpha) z f^{\prime}(z)+\alpha z\left(z f^{\prime}(z)\right)^{\prime}-\left[\alpha z f_{2 j, k}^{\prime}(z)+(1-\alpha) f_{2 j, k}(z)\right] \phi\left(e^{i \theta}\right)\right\} \\
\neq 0 .
\end{aligned}
$$

It is well known that,

$$
z f^{\prime}(z)=f(z) * \frac{z}{(1-z)^{2}} .
$$

From the definition of $f_{2 j, k}(z)$,

$$
f_{2 j, k}(z)=\frac{1}{2}((f * h)(z)+\overline{(f * h)(\bar{z})})
$$

where $h$ is defined by (4.2). Substitute (4.5)and (4.6)in (4.4), we get (4.7).

Theorem 4.2. Let $f \in \mathcal{A} \phi \in \mathcal{P}$. Then $f \in \mathcal{C}_{p}^{j, k}(\alpha, \phi)$ if and only if

$$
\begin{aligned}
\frac{1}{z p}\{f * z[(1-\alpha) & \left.\left.\left(\frac{z}{(1-z)^{2}}-\frac{\phi\left(e^{i \theta}\right)}{2} h\right)+\alpha z\left(\frac{z}{(1-z)^{2}}-\frac{\phi\left(e^{i \theta}\right)}{2} h\right)\right]^{\prime}(z)\right\} \\
& -\frac{1}{z p}\left\{\phi\left(e^{i \theta}\right) \cdot f *\left[z\left(\frac{1-\alpha}{2} h+\frac{\alpha z}{2} h^{\prime}\right)\right](\bar{z})\right\} \neq 0
\end{aligned}
$$

for all $z \in \mathbb{U}$ and $0 \leq \theta<2 \pi$, where $h$ is given by (4.2).

\section{References}

[1] R.M. Ali, A. O. Badghaish, V. Ravichandran, Multivalent functions with respect to $n$-ply points and symmetric conjugate points, Comput. Math. Appl., 60, No. 11 (2010), 2926-2935.

[2] R. Chandrashekar, Rosihan M. Ali, See Keong Lee, V. Ravichandran, Convolutions of meromorphic multivalent functions with respect to $n$-ply points and symmetric conjugate points, Appl. Math. Comput., 218, No. 3 (2011), 723-728, doi: 10.1016/j.amc.2011.03.069.

[3] Paul Eenigenburg, Sanford S. Miller, Petru T. Mocanu, Maxwell O. Reade, On a Briot-Bouquet Differential Subordination. General Inequalities, 3 (Oberwolfach, 1981), 339-348, Internat. Schriftenreihe Numer. Math., 64, Birkhäuser, Basel, 1983. 
[4] R.Md. El-Ashwah, D.K. Thomas, Some subclasses of close-to-convex functions, J. Ramanujan Math. Soc., 2, No. 1 (1987), 85-100.

[5] A.W. Goodman, Univalent Functions, Volume I, Mariner, Tampa, FL, 1983.

[6] Ian Graham, Gabriela Kohr, Geometric Function Theory in One and Higher Dimensions, Monographs and Textbooks in Pure and Applied Mathematics, 255. Marcel Dekker, Inc., New York, 2003.

[7] P. Liczberski, J. Połubiński, On $(j, k)$-symmetrical functions, Math. Bohem., 120, No. 1 (1995), 13-28.

[8] Wan Cang Ma, David Minda, A unified treatment of some special classes of univalent functions, In: Proceedings of the Conference on Complex Analysis (Tianjin, 1992), 157-169, Conf. Proc. Lecture Notes Anal., I, Int. Press, Cambridge, MA, 1994.

[9] K.S. Padmanabhan, R. Parvatham, Some applications of differential subordination, Bull. Austral. Math. Soc., 32, No. 3 (1985), 321-330.

[10] R. Parvatham, S. Radha, On $\alpha$-starlike and $\alpha$-close-to-convex functions with respect to $n$-symmetric points, Indian J. Pure Appl. Math., 17, No. 9 (1986), 1114-1122.

[11] K. Sakaguchi, On a certain univalent mapping, J. Math. Soc. Japan, 11 (1959), 72-75, doi: 10.2969/jmsj/01110072.

[12] C. Selvaraj, K.R. Karthikeyan, G. Thirupathi, Multivalent Functions with respect to symmetric conjugate points, Punjab University Journal of Mathematics, 46, No. 1 (2014), 1-8.

[13] Z.-G. Wang, C.-Y. Gao, On starlike and convex functions with respect to $2 k$-symmetric conjugate points, Tamsui Oxf. J. Math. Sci., 24, No. 3 (2008), 277-287.

[14] Z.-G. Wang, C.-Y. Gao, S.-M. Yuan, On certain subclasses of close-toconvex and quasi-convex functions with respect to $k$-symmetric points, $J$. Math. Anal. Appl., 322, No. 1 (2006), 97-106.

[15] Z.-G. Wang, Y.-P. Jiang, H.M. Srivastava, Some subclasses of multivalent analytic functions involving the Dziok-Srivastava operator, Integral Transforms Spec. Funct., 19, No-s: 1-2 (2008), 129-146. 
[16] S.-M. Yuan, Z.-M. Liu, Some properties of $\alpha$-convex and $\alpha$-quasiconvex functions with respect to $n$-symmetric points, Appl. Math. Comput., 188, No. 2 (2007), 1142-1150. 
